\title{
Lymphatic vessel contractile activity and intestinal inflammation
}

\section{Theresa F Wu/*, Wallace K MacNaughton, Pierre-Yves von der Weid/*/+}

\author{
Mucosal Inflammation Research Group *Smooth Muscle Research Group, Department of Physiology and Biophysics, Faculty \\ of Medicine, University of Calgary, 3330 Hospital Drive N.W., Calgary, T2N4N1, Alberta, Canada
}

\begin{abstract}
Edema is a consistent observation in inflamatory bowel disease (IBD), and immune responses are inevitable in inflammation. Because the lymphatic system is an integral part of both tissue fluid homeostasis and immune reactions, it is likely that lymphatics play a role in the complex etiology of IBD. Despite the consistent findings that the lymphatic system is altered during gastrointestinal inflammation, the majority of studies conducted on the disease only mention the lymphatic system in passing. The effects of inflammatory mediators on lymphatic vessel function also remain poorly defined, despite its essential role in immunity and prevention of tissue edema. Processes allowing effective lymph transport are altered during inflammation, however, the mode of alteration and reason why lymphatics are ineffective in inflammatory reactions need to be further investigated. In addition, these processes have not yet been examined in an appropriate animal model and little has been done using in vivo methods of investigation in any model of gastrointestinal inflammation. This paper reviews the role of the lymphatic system in intestinal inflammation, as well as the role of the inflammatory products in mediating lymphatic contractile function.
\end{abstract}

Key words: lymphatic vessel - lymphatic pumping - inflammation - Crohn's disease - prostanoids - edema

The lymphatic system plays an essential role in the maintenance of tissue homeostasis, removing interstitial fluid and macromolecules from extracellular spaces and returning them back to the blood circulation, thereby preventing edema. The system is also a fundamental component of the immune response, transporting antigens and antigen-presenting cells to lymph nodes where immune cells can be activated, and providing a path for immune competent cells to return to the blood stream. An essential property of lymphatic vessels - conferring the ability to perform these functions - is their capacity to exhibit spontaneous rhythmical constrictions.

The lymphatic contractile function is particularly important to the resolution of inflammation-associated edema, where vessel pumping must help offset increase in interstitial fluid resulting from increases in vascular permeability. A common condition associated with the inflammatory bowel diseases (IBD), Crohn's disease (CD) and ulcerative colitis (UC), is submucosal edema; this has been a consistent observation since the first accurate description of CD, and has been described as one of the essential histological features of the disease (Robb-Smith 1971). Examination by Heatley (1980) of human patients undergoing surgery for CD was one of many investigations demonstrating mesenteric lymphatic obstruction during inflammatory processes. In fact, lymphatic obstruction and dilation are frequently observed in IBD, suggesting impaired function and poor drainage of extracellular fluid, proteins, and other macromolecules. A consequence of interstitial edema is the accumulation of

Financial support: Crohn's and Colitis Foundation of Canada ${ }^{+}$Corresponding author. E-mail: vonderwe@ ucalgary.ca Received 8 November 2004

Accepted 30 December 2004 dead cells and bacteria, which can cause tissue hypoxia and fibrosis. It is this dense fibrosis and muscular hypertrophy that determines the stenosis and strictures that can occur in IBD (Robb-Smith 1971). Thus, lymphatic circulation may play an imperative role in IBD, as impaired function may actively participate in the delayed immmunological response, exacerbate microbial infections, and hinder the prompt resolution of inflammation-associated edema.

\section{The lymphatic system}

Lymphatic vessels serve as a drainage system for the pool of fluid that collects in the interstitium. This interstitial fluid, along with various molecular and cellular components, enters the lymphatic system through blindended initial lymphatics, while bicuspid valves within the lymphatic vessels promote the flow of fluid into the collecting vessels by preventing backflow. Initial lymphatics empty their contents into collecting lymphatics, which have layers of smooth muscle in their wall that are responsible for spontaneous vessel constrictions (Ryan 1989). The phasic contractions of the smooth muscle layer cause a transient compression of the chambers, propelling lymph, macromolecules, antigenic substances, and/or lipids through the one-way valves into the downstream chambers in a pulsatile manner. The ability of the lymphatic vessels to exhibit spontaneous phasic constrictions is the mechanism by which the system performs its essential functions.

The lymphatic system is especially important in inflammatory conditions, where vasodilation and an increase in vascular permeability occur in response to a release of inflammatory mediators due to trauma to the body. This results in an increase of plasma fluid and protein leakage into the interstitial space. These changes in vascular permeability must be offset by increases in lymphatic vessel function. However, efficient reuptake does not always occur, resulting in edema, a cardinal sign of inflammation. 
Although lymphatic vessels have an intrinsic ability to constrict spontaneously, smooth muscle contractility is subject to regulation by various physiological factors. Changes in the interstitial and intraluminal environment occur frequently, and so lymphatic vessels must adapt in changing their behaviour. Lymphatic vessels have the ability to modulate their contractile function in response to factors such as mechanical, endothelial, neural, hormonal, and humoral, factors.

\section{Prostanoids and lymphatic vessels}

Arachidonic acid and its metabolites are among the most important mediators of inflammatory reactions. Importantly, these substances have been shown to directly act on lymphatic vessels and are important modulators of lymphatic function (Johnston 1987). Because of the numerous metabolites that can be produced from arachidonic acid, response of lymphatics to arachidonic acid itself is variable, depending on the predominant metabolite into which it is converted; some are inhibitory, while others are excitatory.

Early studies on cyclooxygenase (COX) inhibitors and inhibitors of other pathways of arachidonate metabolism showed that spontaneous lymphatic pumping was abolished when these metabolic participants were repressed (Johnston \& Gordon 1981). These studies also showed that application of some leukotrienes as well as a prostaglandin $\mathrm{H}_{2}$ /thromboxane $\mathrm{A}_{2}\left(\mathrm{PGH}_{2} / \mathrm{TXA}_{2}\right)$ mimetic induced rhythmic constriction in non-contracting vessels. In a study on rat iliac lymphatics, arachidonic acid caused a significant decrease in vessel diameter via production of constrictor prostaglandins (Mizuno et al. 1998). These effects were blocked by indomethacin, suggesting that these metabolites were produced through COX. The constrictions caused by arachidonic acid were converted to dilations when $\mathrm{PGH}_{2} / \mathrm{TXA}_{2}$ receptors were blocked by antagonists. In the same study, $\mathrm{PGE}_{2}$ caused a dilation in lymphatic vessels that was unaffected by indomethacin (Mizuno et al. 1998). When the endothelial layer was removed from the lymphatic vessel, response to arachidonic acid was significantly reduced, suggesting that the endothelium plays a role in its production, but that other non-endothelial cells may also release prostaglandins. Substance P and ATP were shown to enhance the rate of constriction in guinea-pig mesenteric lymphatics. Attenuation of this increase by indomethacin and SQ29548 suggested that prostanoid $\mathrm{PGH}_{2} / \mathrm{TXA}_{2}$ released from the endothelium is activated by both substance $\mathrm{P}$ and ATP (Rayner \& van Helden 1997, Gao et al. 1999). These studies illustrate the complicated nature by which the lymphatic system responds to its modulators.

\section{Inflammatory bowel disease}

IBD is an ensemble of complex disorders, such as CD and UC, involving chronic inflammation of the gastrointestinal tract. The inflammation is often characterized by periods of flare-ups, with symptoms such as diarrhea, abdominal pain, and cramping followed by resolution or "remission". Current theories about the pathogenesis of IBD point to an impaired mucosal immune response in a susceptible host to the microbes within the intestinal flora. However, the exact mechanisms of immune, environmental, and genetic involvement remain poorly understood.

IBD has variable expressions with a multitude of morphological appearances. CD can affect any part of the gastrointestinal tract from the mouth to the anus, and is characterized by patches of inflammation with intermittent areas of healthy tissue. The inflammation extends through all layers of the gastrointestinal wall. UC-associated inflammation typically begins in the rectum and extends proximally and abruptly stops. It is superficial, rarely penetrating beyond the mucosa. In contrast to $\mathrm{CD}$, the rectum is almost always involved, and the inflammation is continuous and uniform with no skip lesions.

Vascular abnormalities are common in the pathology of IBD, as microvascular compromise is a common precursor to tissue damage. It is believed that the increase in vascular permeability, resultant edema and local ischaemia may perpetuate the disease (Allison 1998). These characterizing features of IBD - submucosal edema, inflammation, and changes in microvasculature suggest the involvement of the lymphatic system in disease resolution, as lymphatics are intimately linked to each of these altered physiological functions.

Current therapy for IBD includes surgical intervention, nutritional therapy, as well as use of drugs such as corticosteroids, immunosuppressants, and COX inhibitors, among others. Of particular interest are the COX inhibitors, as the arachidonic acid derivatives are inflammatory mediators that have potent effects on lymphatic vessel function.

\section{Prostanoids and IBD}

Following an enormous amount of research into the role of prostagandins in mucosal defense, it has been well established that suppression of COX leads to gastrointestinal ulceration associated with the use of nonsteroidal anti-inflammatory drugs (Vane 1971). It has been shown that prostaglandins exert a cytoprotective role by maintaining mucosal blood flow, enhancing epithelial resistance to cytotoxin injury, and by enhancing secretion of mucous and bicarbonate ion in the gastrointestinal tract (Hawkey \& Rampton 1985). Many studies have also shown that inhibition of COX may exacerbate mucosal injury. For example, administration of a COX-2 selective inhibitor to rats with gastric ulcers or colitis led to an intensified inflammation and inhibition of ulcer healing (Reuter et al. 1998).

It has been hypothesized that prostaglandins can down-regulate inflammatory responses and limit the severity of mucosal injury by inhibiting the function of inflammatory cells within the mucosa. For example, Kunkel et al. (1988) demonstrated that prostaglandin $\mathrm{E}_{2}$ is a potent suppressor of tumor necrosis factor (TNF)- $\alpha$ gene expression in macrophages. However, inhibition of COX with non-steroidal anti-inflammatory drugs increased the release of TNF- $\alpha$ from macrophages (Santucci et al. 1994). Because COX metabolites clearly have a significant effect on intestinal inflammation, and have been shown to have direct effects on lymphatic function, it follows that COX metabolites should exert their effects on the lymphatic system during IBD. These effects are important with respect to the ability of lymphatic vessels to reduce the 
inflammation-associated edema and to the role that the inflammatory mediators play in IBD.

\section{The role of lymphatics in IBD}

One of the most consistent pathological features observed in patients suffering CD and UC is lymphatic obstruction and submucosal edema leading to extensive dilation of the lacteals. Pathologists have noted for decades that marked edema and engorgement of capillaries and lymphatics are one of the first morphological changes that occur in intestinal inflammation (Robb-Smith 1971). An early study, in 1936, by Reichert and Mathes demonstrated that injecting sclerosing agents into canine mesenteric lymphatics resulted in granulomatous enteritis. A similar experiment was conducted years later, demonstrating that injection of formalin into porcine mesenteric lymph nodes resulted in mucosal ulcerations and subserosal fibrosis similar to those seen in regional enteritis (Kalima 1976). In a study on normal and diseased human bowel, patients who were undergoing surgery for $\mathrm{CD}$ were studied to determine the efficiency of lymphatic drainage. It was found that affected, and some apparently unaffected areas of the mesentery showed a significant level of lymphatic failure. Further examination of unaffected areas with lymphatic obstruction confirmed the presence of early IBD (Heatley et al. 1980).

Due to the increase in vascular permeability and resultant increase in interstitial fluid, lymph flow is generally believed to increase during inflammatory reactions. The lymphatic system must thus play a crucial role in edema resolution during IBD (Kirsner \& Shorter 1975). It has been shown that mesenteric lymphatic pumping is increased during edemagenic stress caused by dilution of plasma in rats in vivo (Benoit et al. 1989). This was due to the increase in distension of the lymphatic wall, which may be the situation when lymphatics are overloaded in cases of edema.

Although the involvement of lymphatic vessels was demonstrated in these earlier studies, relationship between lymphatics and IBD was not investigated further in the late 1980s and early 1990s. It was not until recently that investigations on lymphatic vessels in the pathogenesis of IBD began again in earnest, especially in the area of initial lymphatics. Mooney et al. demonstrated in 1995 that a significant proportion of granulomas seen in patients with $\mathrm{CD}$ was associated with initial lymphatic vessels and that blood vessel involvement was a secondary rather than primary phenomenon. This finding led the authors to suggest that "granulomatous lymphangitis is a primary lesion of Crohn's disease, and the consequence of the localisation of granulomatous inflammation is the submucosal edema and fibrosis which gives rise to many of the ...histological features of the disease" (Mooney et al. 1995). The authors further hypothesized that the antigens that cause CD may be taken up by macrophages, which then enter the lymphatic system. In 2003, two studies demonstrated a proliferation of initial lymphatics in all areas of the colonic mucosa of patients with UC (Kaiserling et al. 2003) and in the colonic and ileal mucosa of patients with UC and CD respectively (Geleff et al. 2003). A year later, it was shown that lym- phatic capillaries in the colon, which are normally distributed beneath the muscularis mucosa, proliferate into the lamina propria and submucosa in patients with chronic $\mathrm{UC}$, and that this association was proportional to the severity of the disease. In addition, this study showed that the integrity of the lamina propria, in regards to lymphatic distribution, was restored with disease resolution (Fogt et al. 2004). In a study examining the role of collecting lymphatics in IBD, Tonelli (2000) suggested that CD may be caused by a congenital lack of mesenteric lymphatic collectors, causing lymph stasis and lymphangitis, and gastrointestinal inflammation due to the inability to take up and remove toxic bacterial substances. Although this is an extreme view, this study lends support to the hypothesis that the lymphatic system plays a role in the development of IBD.

The aforementioned studies suggest that intervention at the level of the lymphatic system may serve to ease some of the symptoms that IBD patients suffer. Because increased vascular permeability during inflammation is thought to be caused by release of inflammatory metabolites, they could play a pivotal role in modulating lymphatic vessel function. Although it is known that the lymphatic system is intimately involved in and highly altered during the disease, the exact role of lymphatics is not yet known. Many studies have also been conducted, illustrating the potent effect of inflammatory mediators such as COX metabolites on lymphatic function. Although a failure in the lymphatic system may not be the direct cause of the disease, the inflammation may have a significant effect on normal vessel function, and the inability of the vessels to function normally may exacerbate an already deleterious condition.

\section{Future directions}

Although the involvement of lymphatic blockage in IBD has been observed in humans and in animal models (Kalima et al. 1976, Heatley et al. 1980), few studies have investigated the involvement of lymphatic contractile activity in inflammatory reactions, and its role in resolution of IBD remains poorly understood. The effects of inflammatory mediators on lymphatic pathophysiology have not been adequately assessed. In addition, clinical and experimental methods for examining lymphatic vessel function in the gastrointestinal tract and that can be effectively used during IBD are yet to be established.

There is clearly an important role for lymphatic vessels in IBD, and it would be interesting and important to clarify the exact mechanisms of lymphatic changes in these circumstances. It is likely that inflammatory mediators released during $\mathrm{CD}$ and UC alter lymphatic vessel function and impair lymph flow, exacerbating tissue edema and accumulation of dead cells and bacteria. However, this has yet to be demonstrated experimentally. Studies such as these could result in a better understanding of multifactorial intestinal disorders, and may provide new understanding of the complex tissue reactions occurring during the inflammatory response. By gaining an understanding of lymphatic pumping regulation and its mediation by inflammatory metabolites, therapeutic agents may be designed that assist in the reduction of edema 
and aid in a more efficient resolution of IBD. Understanding the processes involved in, and altered by, IBD will help us in the comprehension of how to treat some of the debilitating symptoms of CD and UC.

\section{REFERENCES}

Allison MC, Dhillon AP, Lewis WG, Pounder RE 1998. Inflammatory Bowel Disease, Mosby International Limited, Barcelona, Spain.

Benoit JN, Zawieja DC, Goodman AH, Granger HJ 1989. Characterization of intact mesenteric lymphatic pump and its responsiveness to acute edemagenic stress. Am J Physiol 257: H2059-H2069.

Fogt F, Pascha TL, Zhang PJ, Gausas RE, Rahemtuly A, Zimmerman RL 2004. Proliferation of D2-40-espressing intestingal lymphatic vessels in the almina propria in inflammatory bowel disease. Int J Molec Med 13: 211-214.

Gao J, Zhao J, Rayner SE, van Helden DF 1999. Evidence that the ATP-induced increase in vasomotion of guinea pig mesenteric lymphatics involves an endothelium-dependent release of thromboxane $\mathrm{A}_{2}$. Br J Pharmacol 127: 15971602.

Geleff S, Schoppmann SF, Oberhuber G 2003. Increase in podoplanin-expressing intestinal lymphatic vessels in inflammatory bowel disease. Virchows Arch 442: 231-237.

Hawkey CJ, Rampton DS 1985. Prostaglandins and the gastrointestinal mucosa: are they important in its function, disease or treatment? Gastroenterology 89: 1162-1188.

Heatley RV, Bolton PM, Hughes LE, Owen EW 1980. Mesenteric lymphatic obstruction in Crohn's disease. Digestion 20: 307-313.

Johnston MG 1987. Interaction of inflammatory mediators with the lymphatic vessel. Pathol Immunopathol Res 6: 177189.

Johnston MG, Gordon JL 1981. Regulation of lymphatic contractility by arachidonate metabolites. Nature 293: 294297.

Kaiserling E, Krober S, Geleff S 2003. Lymphatic vessels in the colonic mucosa in ulcerative colitis. Lymphology 36: 52-61.

Kalima TV, Salomiemi H, Rahko T 1976. Experimental regional enteritis in pigs. Scand J Gastro 11: 353-362.

Kirsner JB, Shorter RG 1975. Inflammatory Bowel Disease, Lea and Febiger. Philadelphia.

Kunkel SL, Spengler M, May MA, Spengler R, Larrick J, Remick $D$ 1988. Prostaglandin $\mathrm{E}_{2}$ regulates macrophage derived tumor necrosis factor gene expression. J Biochem 263: 53805384.

Mizuno R, Koller A, Kaley G 1998. Regulation of the vasomotor activity of lymph micorvessels by nitric oxide and prostaglandins. Am J Physiol 274: R790-R796.

Mooney EE, Walker J, Hourihane DO 1995. Relation of granulomas to lymphatic vessels in Crohn's Disease. J Clin Pathol 48: 335-338.

Rayner SE, van Helden DF 1997. Evidence that the substance $\mathrm{P}$-induced enhancement of pacemaking in lymphatics of the guinea-pig mesentery occurs through endothelial release of thromboxane $\mathrm{A}_{2}$. Br J Pharmacol 121: 1589-1596.

Reichert FL, Mathes ME 1936. Experimental lymphedema of the intestinal tract and its relation to regional cicatrizing enteritis. Ann Surg 104: 610-616.

Reuter MK, Asfaha S, Buret A, Sharkey KA, Wallace JL 1998. Exacerbation of inflammation-associated colonic injury in rat through inhibition of cyclooxegenase-2. J Clin Invest 98: 2076-2085.

Robb-Smith AHT 1971. Symposium on Crohn's disease. Proc Royal Soc Med 64: 157-167.

Ryan TJ 1989. Structure and function of lymphatics. J Invest Dermatol 93: 18S-23S.

Santucci L, Fiorucci S, Giansanti M, Brunori PM, Dimatteo FN, Morelli A 1994. Petnoxifylline prevents indomethacininduced acute mucosal damage in rats: role of tumour necrosis factor. Gut 35: 909-915.

Tonelli P 2000. New developments in Crohn's disease: solution of doctrinal mysteries and reinstatement as a surgically treatable disease. 1. The process is not a form of enteritis but lymphedema contaminated by intestinal contents. Chir Ital 52: 109-121.

Vane JR 1971. Inhibition of prostaglandin synthesis as a mechanism of action for aspirin-like drugs. Nat New Biol 231: 232-235. 